\title{
Framework for Cloud Computing Platform with a Virtualized Network Infrastructure
}

\author{
Pourya Shamsolmoali \\ Dept .of Computer science \\ Hamdard University \\ New Delhi, India
}

\author{
M.Afshar.Alam \\ Dept. of computer science \\ Hamdard University \\ New Delhi, India
}

\begin{abstract}
After the development of cloud computing technology and internet, manufacture of cloud computing, which is operated independently by the third party, with high scalability and low energy consumed, become a new network manufacturing mode, this paper starts with the cloud Service models overview, and then the paper discusses the usage of cloud computing technology for designing a platform virtually for a group of scientist to display their achievement on internet. The authors compare two kinds of models and introduced architecture for design platform, the platform allows scientists to share their achievement by using the unified portal.
\end{abstract}

Keywords: cloud computing, design platform, virtualization technology

\section{INTRODUCTION}

Cloud computing is a pattern that focus on sharing infrastructure, data and computation over scalable network. Basically cloud is an abstraction for the complex infrastructure.

Cloud is the supplement and evaluation of several concepts [1] with the development of IT and applications, it is very important for Scientists and Scientific department to get and share information. cloud computing can prove to be very useful in this part, the authors observed, many scientists with good ideas and artifacts facilitated through the careful design of assessment elements., many of them share there achievements on the internet by renting virtual host or public server, but some of scientists have limited resources, so many ideas are never shared or published[2] This paper is going to present an efficient, scalable and flexible platform by using the features of virtualization technology for scientists to deploy there achievement.

\section{CLOUD COMPUTING}

Cloud computing is strongly dependent on virtualization technology and services. The IT resources may be virtual IT infrastructure, or some data, or a business system from the perspective of cloud platform. Cloud computing is distributed design modes for the customer. Cloud computing is a collection of all kinds of software and hardware resources, the collection should provide convenient and reliable resources. Cloud computing provides unlimited infrastructure to store and execute customer data and program. Customers do not need to own the infrastructure, they are merely accessing it; they can forego capital expenditure and consume resources as a service paying instead for what they use. [1]

Three main types of service models in cloud computing are as follows:

2.1 Software as a service (Saas): the clients may select for ready customized application, but do not have control over background environment such as operating system, hardware or network parameters (e.g., web-based email).[3]

2.2 Platform as a service (Paas): in this type of services, clients have control over change in application on hosting environment such as system software but Saas does not provide over operating system, hardware and networking parameters (e.g. configurations).[3]

2.3 Infrastructure as a service(Iaas): The capability provided to the consumer is to provision processing, storage, networks, and other fundamental computing resources where the consumer is able to deploy and run arbitrary software, which can include operating systems and applications.(e.g., host firewalls).[1]

\section{VIRTUALIZATION TECHNOLOGY}

With virtualization techniques, multiple operating systems can concurrently run on a single physical system. The resources of one physical computer can be partitioned into logical resources and rearranged into so-called virtual machines or complete computers. The ingredient resources of which are dedicated logical fragments of resources of one or more physical machines [4]. Function together with its required middleware stack and even the required operation system can be hosted by a virtual machine. This results in a significant increase in utilization of even a single physical computer by running various heterogeneous application stacks on the same machine.

\section{PORTAL PLATFORM}

A portal consist of application that deliver information and/or services from multiple sources, via a device specific channel at a device specific location, The Portal system has a very important role, in accordance with the design, a unified portal platform includes three major closely complimented with the innovation. [2] 


\section{SYSTEM ARCHITECTURE}

Fig 1 gives the architectural overview of our cloud computing system.

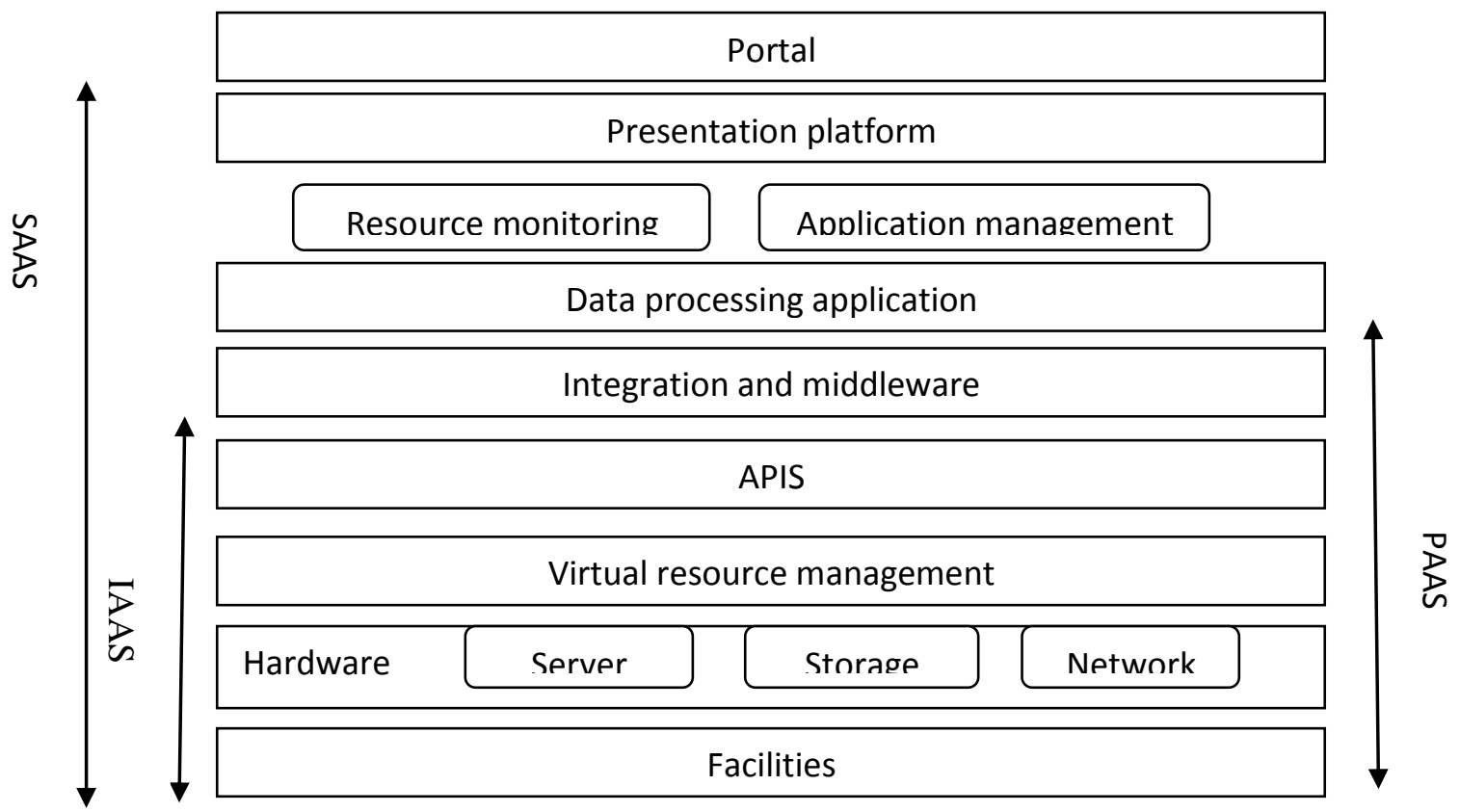

Fig 1.the system architecture

By analyzing the architecture carefully the following aspects can be figured out, at the foundation of this frame work is a data center infrastructure technology architecture, which consist of three salient blocks the network, server and storage. This layer hosts all the services that are delivered to a cloud consumer [5] following the data center is a virtual resource management layer, this layer control the virtual machines function and monitor them. APIs are application programming interface used to build applications in cloud computing market; APIs allow software to request data and computations from one or more services through a direct or indirect interface. Next is a middleware layer that consists of software that provides bridging functionality between separate application or software processes. Integration middleware is used to connect existing applications with functionality from new applications [6]. Data processing layer is use for processing; each working node gets a job description from the data base, retrieves the data, processes it and sends the results to intermediate servers. The framework includes the resources, management layer and server management, the resources management layer mainly provides computing resources management, while the server management layer provides business logic. The topmost layer is the consumer facing layer or portal, this is the layer where service is defined requested, and managed by the consumer, the architecture that monitored above, includes operation, management and security elements.

\section{CHOICE OF VIRTUAL MACHINE}

Citrix Xen and KVM are two mainstream virtualization engines build on the Linux platform, Citrix Xen is an external hypervisor program, and it can control virtual machines and allocate resources to multiple clients,
Citrix Xen supports full virtualization and Para virtualization, it is also an open source virtual machine. Xen currently runs only on x86 architecture [2]. KVM (Kernel-based Virtual Machine) is a part of the Linux kernel, we can use the usual Linux scheduler and memory management, which means KVM, is smaller and easier to be use. And it only works with 64 bit CPU platform, Using $\mathrm{KVM}$; one can run multiple virtual machines running unmodified Linux or Windows images. Each virtual machine has private virtualized hardware: a network card, disk, graphics adapter, etc; structure of KVM is shown in fig 2.

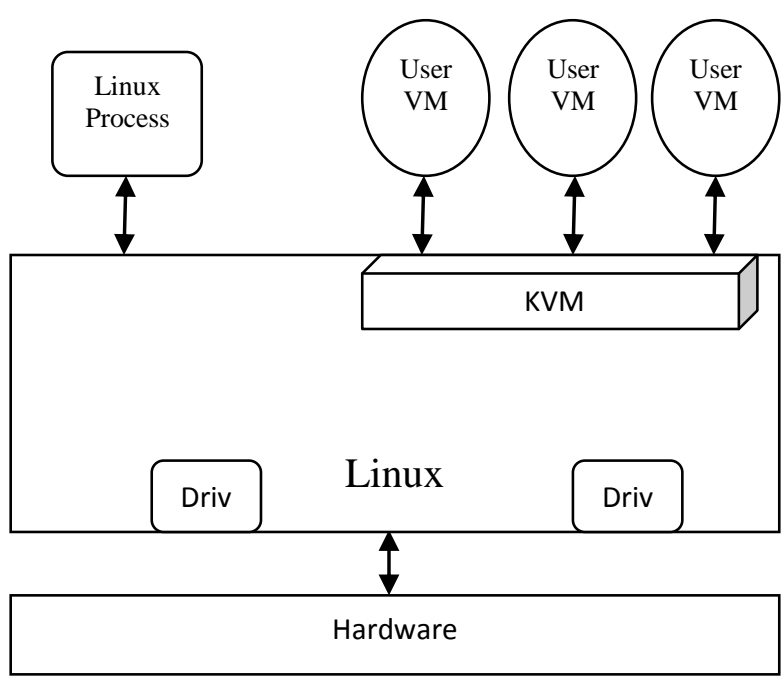

Fig 2.structure of KVM

Gn management parı, n vivı can support Libvirt API programming interface, Lbvirt is a Libvirt to manage local or remote virtual resources. The scope of the libvirt API is intended to extend to all functions necessary for 
deployment and management of virtual machines and allowing support for different underlying virtualization technologies and operating systems [7]. Architecture of Libvert virtualization library is shown in fig 3 .

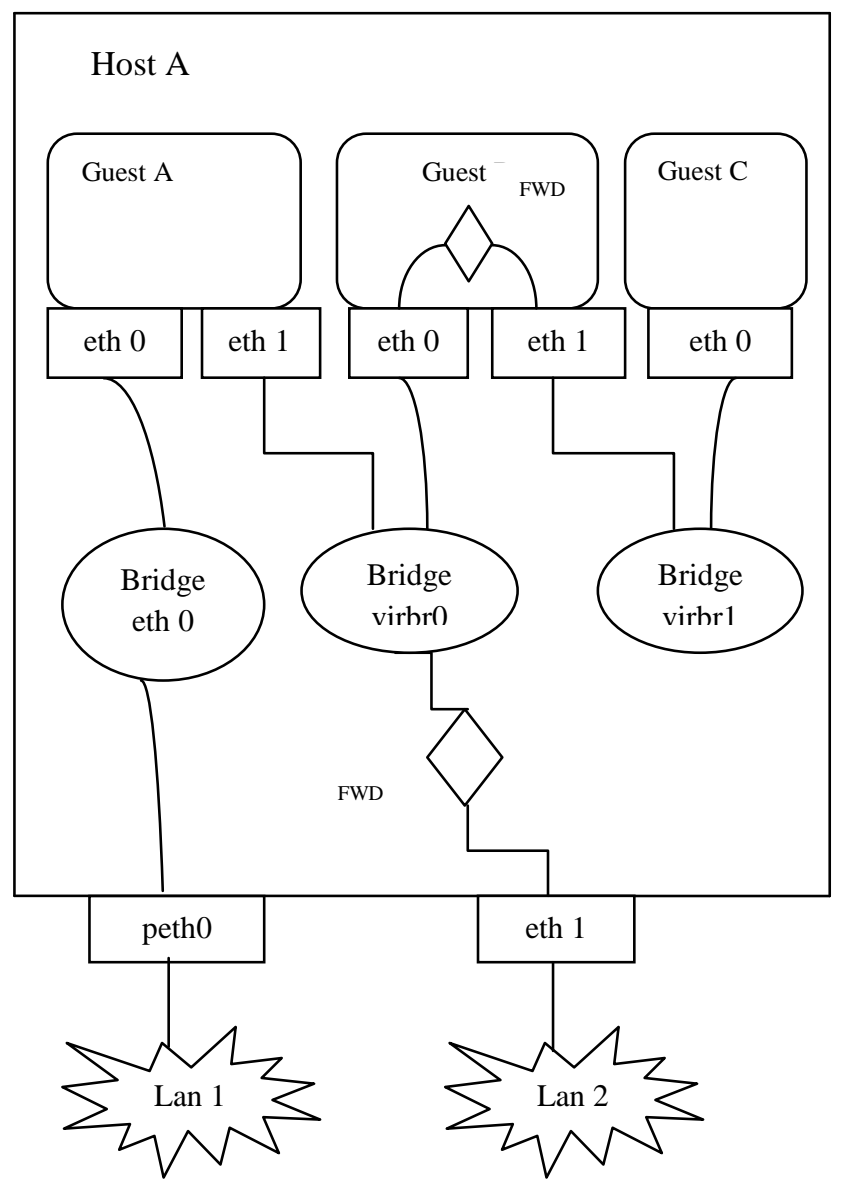

Fig 3. Architecture of Libvert virtualization

\section{1 virtual Resource Scheduling Control}

Virtual resource scheduling includes two parts

a) The resource load scheduling: in the cloud computing platform the load on server fluctuates, either too high or too low, so we can use source load scheduling, to present thresholds, so when server load arrives to the threshold, system will send dynamically a number of virtual machines to the server. If low load will observe or other problems arise a warning will show the administrator, to migrate manually to achieve physical server load balancing.

b) High availability of scheduling: the system will move around the health state of virtual machine, when turned on. When Virtual machine cannot be accessed in the system, it will reorganize the resources to run the virtual machine [8]

\section{ACCESS CONTROL AND MANAGEMENT OF VIRTUAL MACHINE}

As the number of users in the cloud increase, with virtual partitions; it will bring to administration a very large work load, so self management platform has been built, so scientists have self management operation on their own virtual machine. Process of creation a virtual machine is shown in fig 4 .

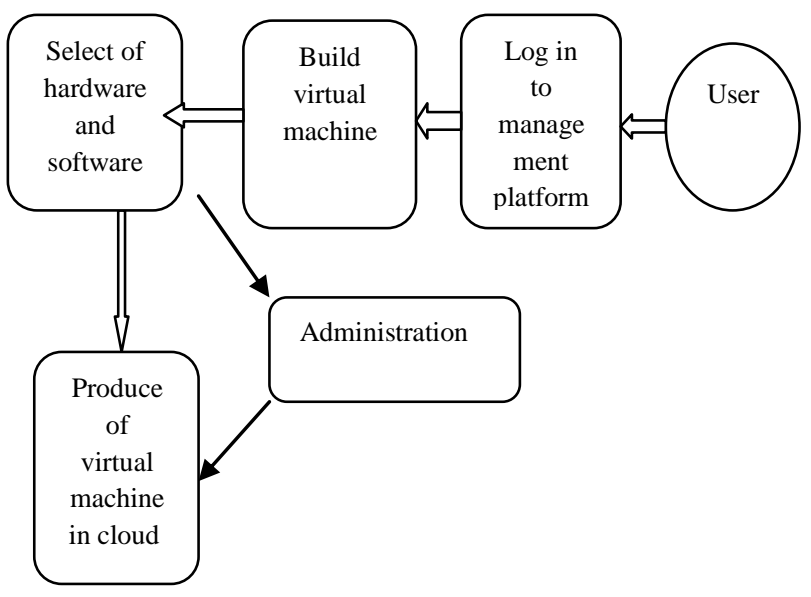

Fig. 4. Process of creation a virtual machine

Self management of the platform it includes two functions:

a) Creation and deletion of virtual machine: after a user $\log$ on, they are presented with a management function to build a virtual machine according to their usages and could select the hardware and software resources. And also when user face that the virtual machine is not required, they are able to delete their virtual machine.

b) Monitor and manage the virtual machine: user can log in and can see the situation of their virtual machine such as CPU and memory, and also can log into the system and manage the resources.

\section{CONCLUSION}

Cloud computing in simple words is about choice. It enables the organization to deliver service and information to consumer. Virtualization technology is the core of cloud computing and it is fundamental technology for building cloud. This paper focuses on designing a platform for scientists where they can easily publish their achievement. Authors used an application of cloud technology to compare different technologies on virtual machine, to build an open virtual cloud management platform. This platform enables scientists to select hardware and software as per there needs from resources and make their own virtual machine. This technology gives user an opportunity for managing the virtual machine. The platform is flexible and provides many kinds of resources to fulfill diverse range of requirements. 


\section{REFERENCES}

[1] Madhubala.R, "An Illustrative study on Cloud Computing", International Journal of Soft Computing and Engineering (IJSCE) ISSN: 22312307, Volume-1, Issue-6, January 2012.

[2] Liu,zhan-heng, Chang-li Zhang and Tim Reynolds, " A Framwork for an University Display Platform By Using Cloud Computing" ,2011 International Conference on Cyber-Enabled Distributed Computing and Knowledge Discovery, 2011 IEEE, pp 424428

[3] Sharma. Rajkumar and Priyesh Kanungo, " An Intelligent Cloud Computing Architecture Supporting e-Governance ", Proceedings of the 17th International Conference on Automation \& Computing, University of Huddersfield, Huddersfield, UK, 10th September 2011 .

[4] smith.R , " Cloud Computing: The Next Revolution in IT " , October 2009 computing in the cloud, volume 52, new York.
[5] Bakshi. Kapil. "Considerations for Cloud Data Centers: Framework,Architecture and Adoption ", 2011 IEEE, IEEEAC paper\#1150, Version 2,

[6] Madhavapeddy. Anil and Satnam Singh," Reconfigurable Data Processing for Clouds ", Computer Laboratory University of Cambridge 15 JJ Thomson Avenue, Cambridge.2010

[7] www.libvirt.org ,"network management architecture", physical diagram.

[8] Ye. Kejiang, Xiaohong Jiang, Dawei Huang, Jianhai Chen, and Bei Wang, "Live Migration of Multiple Virtual Machines with Resource Reservation in Cloud Computing Environments", 2011 IEEE 4th International Conference on Cloud Computing, pp 267274.

[9] Rahman. Omar Abdul, Masaharu Munetomo and Kiyoshi Akama, "Multi-Level Autonomic Architecture for the Management of Virtualized Application Environments in Cloud Platforms ", 2011 IEEE 4th International Conference on Cloud $\begin{array}{llll}\text { Computing, } & \text { pp } & 754 & 755\end{array}$ 